Intropica 14(1): 16 - 23

\title{
Metabolismo de un tramo en la cuenca baja del río Cesar, departamento del Cesar, en una época de sequía, Colombia
}

\section{Metabolism of a stretch in the lower basin of the Cesar River, department of Cesar, in a dry season, Colombia}

\author{
Ana C. De la Parra-Guerra* (1) y Carlos A. García-Alzate링 \\ *Autor de correspondencia: acdelaparra25@gmail.com
}

Recibido: 22 octubre de 2018

Aceptado: 6 de febrero de 2019

Grupo de investigación Biodiversidad del Caribe colombiano, Facultad de Ciencias Básicas, Universidad del Atlántico

\begin{abstract}
Resumen
Palabras clave:

Las funcionalidades de los sistemas acuáticos son el resultado de interacciones biológicas, condiciones fisicoquímicas del agua y el ambiente terrestre. Estas relaciones son afectadas por acciones antrópicas, como deforestación, minería, agricultura, vertimientos de residuos sólidos y líquidos domésticos e industriales, en general prácticas nocivas para los ecosistemas acuáticos. Este trabajo se realizó con el fin de estimar la variación del metabolismo y las variables fisicoquímicas en la cuenca baja del río Cesar durante la época de máximo estiaje. Se determinó el estado trófico de la cuenca baja del ecosistema acuático por observaciones en los cambios del consumo de $\mathrm{O}_{2}$ y acumulación de $\mathrm{CO}_{2}$, y se cuantificaron los procesos de fotosíntesis y respiración. Se realizaron dos muestreos en cuatro estaciones, entre febrero-abril de 2013, durante el periodo seco. Se midieron las variables fisicoquímicas in situ y algunos nutrientes. Se aplicó el protocolo del metabolismo, utilizando el método de Winkler (botellas clara y oscura), con mediciones cada hora durante $24 \mathrm{~h}$ (curva diaria). El metabolismo neto fue de 10 $732,23 \mathrm{O}_{2} / \mathrm{m}^{3}$-h, el oscuro de $-10731,41 \mathrm{O}_{2} / \mathrm{m}^{3}$-h y la producción primaria bruta de toda la comunidad acuática de -10 736,91 $\mathrm{O}_{2} / \mathrm{m}^{3}$-h. Estos resultados sugieren que las variaciones en el metabolismo neto y oscuro, así como la productividad primaria de la comunidad fueron casi nulas. Adicionalmente, el oxígeno producido durante el día fue consumido rápidamente en la noche. Nuestros datos demuestran que el estado trófico de la cuenca baja del río Cesar es eutrófico-heterotrófico. La respiración excede la producción de $\mathrm{O}_{2}$, lo que genera déficit de este y una disminución de la calidad del ecosistema, resultados que se corroboran con los análisis de las variables fisicoquímicas medidas en el sitio.
\end{abstract}

\section{Abstract}

Key words: The functionalities of aquatic systems are the result of biological interactions, the physicochemical conditions of water and the terrestrial environment, these relationships are affected by anthropic actions, such as: deforestation, mining, agriculture, solid waste discharges and domestic and industrial liquids, general harmful practices for aquatic ecosystems. This research was carried out with the purpose of estimating the variation of the metabolism and physicochemical variables in the lower basin of the Cesar River, during the period of maximum dry season, the trophic state of the lower basin of the aquatic ecosystem was determined by observations in the changes of the $\mathrm{O}_{2}$ consumption and $\mathrm{CO}_{2}$ accumulation, the processes of photosynthesis and respiration were quantified. Two samplings were carried out in four stations, between February-April 2013, equivalent to the dry period. Physicochemical variables in situ and some nutrients were measured. The metabolism protocol was applied, using the Winkler method (clear and dark bottles), with measurements every hour for $24 \mathrm{~h}$ (daily curve). The net metabolism was $-10732.23 \mathrm{O}_{2} / \mathrm{m}^{3}-\mathrm{h}$, the dark one of $-10731.410^{2} / \mathrm{m}^{3}$-h and the gross primary production of the entire aquatic community of $-10736.910^{2} / \mathrm{m}^{3}$-h. These results suggest that the variations in the net and dark metabolism, as well as the primary productivity of the community were almost nil. Additionally, the oxygen produced during the day was consumed quickly at night. Our data show that the trophic state of the lower basin of the Cesar River is eutrophic-heterotrophic, the respiration exceeds the production of $\mathrm{O}_{2}$, which generates deficit of the same and a decrease in the quality of this ecosystem, results that are corroborated with the analyzes of the physicochemical variables measured at the site. 


\section{Introducción}

En los ecosistemas acuáticos, los resultados de las interacciones biológicas junto con las condiciones fisicoquímicas del agua y el medio terrestre son de importancia para comprender sus funcionalidades (Roldán y Ramírez, 2008). En los últimos años, las fuentes hídricas han sido sometidas a numerosas perturbaciones, tales como actividades de deforestación, minería, agricultura, ganadería, vertimientos de residuos sólidos y líquidos de origen doméstico e industrial, los cuales son fuentes de contaminación, además de ser las principales causas de cambios en la estructura de las comunidades biológicas existentes (García-Alzate et al., 2007; 2008; De la Parra et al., 2017). Para conocer los deterioros que inducen dichas perturbaciones sobre los cuerpos de agua, se han empleado diversas medidas de monitorización, en las variables fisicoquímicas, microbiológicas, nutrientes, entre otras. Sin embargo, hasta la fecha los estudios asociados al metabolismo han sido poco considerados, siendo una variable de gran importancia, ya que permiten conocer los cambios que ocurren entre las variaciones de producción/consumo de $\mathrm{O}_{2}$ y $\mathrm{CO}_{2}$ en el agua. Por lo tanto, el metabolismo es considerado un parámetro vital para estimar el estado trófico de los sistemas acuáticos.

El metabolismo se define como la suma de todas las reacciones químicas que se producen en los ecosistemas acuáticos de tipo lénticos y lóticos, que usa como variable global el consumo de oxígeno (Mulholland et al., 2001). Sin embargo, el metabolismo es altamente sensible a cambios en la cobertura de ribera y estrés hídrico (Bunn et al., 1999), debido al consumo de $\mathrm{O}_{2}$ y liberación de $\mathrm{CO}_{2}$ (Dodds 2006; 2007; Dodds y Cole 2007; García-Alzate et al., 2008).

Los análisis de metabolismo son indicadores importantes de autotrofía (lo que se produce en el día no es consumido totalmente en la noche) y heterotrofia (lo que se produce en el día es consumido en la noche) en cuerpos de agua, que pueden ser productores o consumidores netos de materia orgánica (Roldán y Ramírez, 2008). Actualmente, los estudios sobre el metabolismo y la productividad primaria en los ríos tropicales de Colombia son escasos y los pocos que existen son en ríos de alta montaña (Ramírez, 2001; Montoya y Aguirre, 2009; 2013; Donato et al., 2014).

Considerando lo anterior, en el presente trabajo se determinó el metabolismo y las variables fisicoquímicas de la cuenca baja del río Cesar, durante la época seca (período de máximo estrés hídrico), con el fin de clasificar el estado trófico de la cuenca baja, de gran importancia para la población de Valledupar y los municipios, en el 17 departamento del Cesar, Colombia.

\section{Materiales y métodos}

\section{Área de estudio}

La investigación se llevó a cabo en cuatro estaciones (E1, E2, E3 y E4) en la cuenca baja del río Cesar, distribuidas desde el río Surivaca hasta la confluencia de la quebrada Pata de Vaca y Caño de Hoyo (figura 1). La cuenca presenta un régimen pluviométrico de tipo ecuatorial bimodal con un período de altas lluvias y bajas lluvias. La época seca está marcada para los meses enero-marzo y julio-agosto (IDEAM, 2006). Los dos muestreos correspondieron al período seco del año 2013.

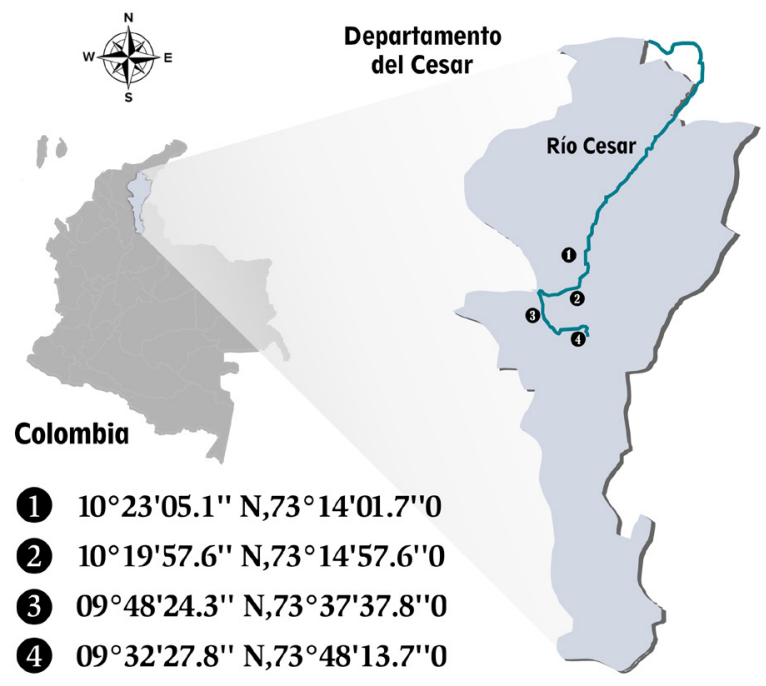

Figura 1. Mapa ubicación del departamento del Cesar, referenciándose las estaciones y coordenadas de muestreo en la cuenca baja del río Cesar.

\section{Fase de Campo}

Variables fisicoquímicas. Se midieron in situ variables como el pH, con un potenciómetro WTW 3210, precisión de 0,001; el oxígeno disuelto, (mg/L), la temperatura del agua y el porcentaje de saturación de oxígeno disuelto, con un Oxímetro WTW 3205, precisión de 0,001; la conductividad $(\mu \mathrm{S} / \mathrm{cm})$, con un Conductímetro WTW 3130 con precisión de 0,001; el caudal con un Flujómetro, y la turbidez con un Turbidímetro HACH-2100 Q. Se tomaron muestras de agua en frascos de vidrio de $250 \mathrm{~mL}$, llevadas inmediatamente en cavas de Icopor refrigeradas al laboratorio de química de la Universidad del Atlántico para los análisis de nutrientes: nitritos $\left(\mathrm{NO}_{2}-\mathrm{N}\right)$, nitratos $\left(\mathrm{NO}_{3}-\mathrm{N}\right)$, nitrógeno total $(\mathrm{NT})$, fosfatos $\left(\mathrm{PO}_{4}-\mathrm{P}\right)$, Demanda Biológica de Oxígeno $\left(\mathrm{DBO}_{5}\right)$ y 
sólidos suspendidos totales (SST), siguiendo las técnicas descritas y recomendadas por el APHA (2005).

Metabolismo. El metabolismo se calculó por el método de Winkler (botella clara y botella oscura; Wetzel y Likens, 2000; Gutiérrez, 2006). Los valores de oxígeno disuelto (OD), porcentaje de saturación de oxígeno disuelto (\% OD), $\mathrm{pH}$ y conductividad fueron medidos cada hora, durante $24 \mathrm{~h}$, con el fin de diseñar una curva del déficit de oxígeno (curva diaria), la cual se realizó en la estación 2.

Clorofila-a (Cl-a). Adicionalmente, se recolectaron muestras de fitoperifiton, para determinar Cl-a. Las muestras se envasaron en frascos oscuros forrados con papel aluminio para su conservación y llevadas refrigeradas al laboratorio de química de la Universidad del Atlántico, en la ciudad de Barranquilla, Colombia.

\section{Fase de laboratorio}

Variables fisicoquímicas. Se midieron nitritos, nitratos, nitrógeno y fosfatos por espectrofotometría (Genesis-5) y $\mathrm{DBO}_{5}$ (incubación cinco días a $25^{\circ} \mathrm{C}$ ), siguiendo las técnicas del APHA (2005).

Metabolismo. Se estimó de acuerdo con la metodología de Wetzel y Likens (2000) y por la ecuación modificada por García-Alzate et al. (2010): Q = p-r $\pm d+a$. Dónde: $\mathrm{Q}=\%$ de cambios de oxígeno en $\mathrm{g} \mathrm{O}_{2} / \mathrm{m}^{3}-\mathrm{h}, \mathrm{p}=\%$ de productividad primaria bruta, $\mathrm{r}=\%$ de respiración, $\mathrm{d}=$ $\%$ de difusión de oxígeno a través de la interfaz aire-agua y a $=\%$ de acumulación del drenaje. La corrección para la difusión o re-aireación d se estimó a través del promedio del déficit de oxígeno que cubre la cuenca baja del río como sitio de estudio. La medida del déficit de oxígeno es entonces multiplicada por una constante de difusión $\mathrm{K}=0$,9. El metabolismo se estimó con la diferencia de los datos de la estación elegida. Metabolismo neto = cambio del DO luz-Difusión. Metabolismo oscuro = cambio del DO oscuro-Difusión. Productividad primaria bruta de la comunidad $(\mathrm{GPP})=$ Metabolismo neto respiración luz. Respiración de la comunidad (CR24) = promedio día noche, respiración escala 24 h (Dodds, 2006; 2007; Dodds y Cole, 2007; García-Alzate et al., 2008; García-Alzate et al., 2010).

Clorofila a (Cl-a). La determinación de $\mathrm{Cl}$-a del fitoperifiton, se realizó por el método espectrofotométrico (T80 + UV/ VIS, Spectrometer PG Instruments) propuesto por APHA (2005), realizándose un filtrado al vacío con filtros de microfibra de vidrio (GF/C- Glass Fiber Filter) de $47 \mathrm{~mm}$ de diámetro. La extracción de los pigmentos se hizo con solución de acetona al 90 \%. Durante este proceso se realizó una maceración mecánica de los filtros en tubos de $15 \mathrm{~mL}$ con $5 \mathrm{~mL}$ acetona; se mantuvo el montaje a $4^{\circ} \mathrm{C}$ durante 24 h y finalmente se sometió a centrifugación durante $15 \mathrm{~min}$ a $3000 \mathrm{rpm}$. Se midió la absorbancia longitudes de ondas (750; 665; 664; 647; 630; 610). Todo el proceso se realizó con luz tenue para proteger las muestras de interferencia. Esta técnica fue hecha en el laboratorio Museo Colecciones Biológicas de la Universidad del Atlántico.

Análisis de los datos. Los datos son representados por medias aritméticas como medida de tendencia central; se calculó el coeficiente de variación (CV) y la desviación estándar (DE) como medidas de dispersión relativa, acompañada de la amplitud (valor máximo y mínimo). Se comprobaron los supuestos de homogeneidad de varianza (Prueba de Bartlett) y prueba de normalidad de los datos (Bondad de ajuste de Kolmogorov-Smirnov: K-S), lo cual arrojaron que los datos no poseen una distribución no normal $(p>0,05)$. Por consiguiente, las comparaciones estadísticas se desarrollaron con base en el análisis no paramétrico de Kruskal-Wallis: K-W. Además, para el análisis del metabolismo, se calculó el déficit de oxígeno (DO = Oxígeno final - Oxígeno inicial): tanto para la botella clara como para la oscura se midió producción y consumo del mismo. Los análisis se realizaron con ayuda de los Software estadísticos de PAST 3.0 (Hammer et al., 2001).

\section{Resultados}

\section{Variables fisicoquímicas}

La temperatura del agua osciló entre $27,40{ }^{\circ} \mathrm{C}$ y 31,80 ${ }^{\circ} \mathrm{C}$; promedio $=29,58{ }^{\circ} \mathrm{C}$. El OD fue bajo en general (promedio $=4,66 \mathrm{mg} / \mathrm{L}$ ). El $\mathrm{pH}$ fue básico con valores entre 7,3 a 8,1 respectivamente. Las saturaciones del \% DO fueron bajas, entre $16 \%$ y $105 \%$ (promedio $=69,50 \%$; DE $=26,88 ; \mathrm{CV}=1039,67$ ). La turbidez (promedio $=108,05$ $\mathrm{NTU}$; DE $=73,03 ; \mathrm{CV}=4899,65$ ) y los SST (promedio = $125,96 \mathrm{mg} / \mathrm{L} ; \mathrm{DE}=70,52 ; \mathrm{CV}=3948,12$ ) fueron variables. La conductividad general fue alta (promedio $=333,00 \mu \mathrm{S}$ / $\mathrm{cm} ; \mathrm{DE}=89,84 ; \mathrm{CV}=2423,94)$.

El caudal disminuyó en la época seca (promedio $=36,24$ $\mathrm{m}^{3} / \mathrm{s} ; \mathrm{DE}=11,45 ; \mathrm{CV}=361,50$ ) (tabla 1 ). Con respecto a los nutrientes, se observaron bajas concentraciones durante los muestreos: el nitrito presentó un promedio $=2,10 \mu \mathrm{g} / \mathrm{L} ; \mathrm{DE}$ $=2,16 ; \mathrm{CV}=223,01$, el nitrato un promedio $=29,35 \mu \mathrm{g} / \mathrm{L}$; $\mathrm{DE}=21,24 ; \mathrm{CV}=1581,1$ con mayores concentraciones en la E1, tendencia similar observada para el nitrógeno (promedio $=31,46 \mu \mathrm{g} / \mathrm{L} ; \mathrm{DE}=21,0 ; \mathrm{CV}=1408,6 \mathrm{y}$ los fosfatos (promedio $=209,44 \mu \mathrm{g} / \mathrm{L} ; \mathrm{DE}=58,92 ; \mathrm{CV}=1657,7$ ). La $\mathrm{DBO}_{5}$ presentó los mayores registros en esta época con un promedio $=11,18 \mathrm{mg} / \mathrm{L} ; \mathrm{DE}=7,24 ; \mathrm{CV}=465,1$ (tabla 1 ). 
Tabla 1. Estadística descriptiva de las variables fisicoquímicas medidas en la cuenca baja del río Cesar; mínimo (MIN), máximo (MÁX), media geométrica (MG), promedio (X), varianza (VA), desviación estándar (DE) y coeficiente de variación (CV).

\begin{tabular}{lrrrrrrr}
\hline Variables & MIN & MÁX & MG & X & VA & DE & CV \\
\hline Temperatura del agua $\left({ }^{\circ} \mathrm{C}\right)$ & 27,4 & 31,8 & 29,5 & 29,6 & 3,7 & 1,9 & 12,4 \\
Oxígeno disuelto $(\mathrm{mg} / \mathrm{L})$ & 3,4 & 6,4 & 4,6 & 4,7 & 1,3 & 1,1 & 27,1 \\
Porcentaje de saturación de oxígeno (\%) & 16,0 & 105,0 & 62,1 & 69,5 & 722,6 & 26,9 & 1039,7 \\
pH & 7,4 & 8,1 & 7,8 & 7,8 & 0,1 & 0,4 & 1,6 \\
Conductividad $(\mu \mathrm{S} / \mathrm{cm})$ & 262,0 & 447,0 & 323,1 & 333,0 & 8071,7 & 89,8 & 2423,9 \\
Sólidos suspendidos totales $(\mathrm{mg} / \mathrm{L})$ & 8,7 & 188,6 & 92,6 & 126,0 & 4973,2 & 70,5 & 3948,1 \\
Caudal $\left(\mathrm{m}^{3} / \mathrm{s}\right)$ & 22,9 & 58,4 & 34,8 & 36,2 & 131,0 & 11,5 & 361,5 \\
Turbidez $(\mathrm{NTU})$ & 9,7 & 193,0 & 70,3 & 108,9 & 5333,1 & 73,0 & 4899,7 \\
Nitrito $\left(\mathrm{NO}_{2}-\mathrm{N}\right)(\mu \mathrm{g} / \mathrm{L})$ & 0,1 & 6,1 & 1,0 & 2,1 & 4,7 & 2,2 & 223,0 \\
Nitrato $\left(\mathrm{NO}_{3}-\mathrm{N}\right)(\mu \mathrm{g} / \mathrm{L})$ & 0,0 & 73,8 & 8,2 & 29,4 & 464,1 & 21,5 & 1581,2 \\
Nitrógeno $\left(\mathrm{N}_{2}\right)(\mu \mathrm{g} / \mathrm{L})$ & 0,5 & 73,9 & 19,3 & 31,5 & 443,1 & 21,1 & 1408,6 \\
Fosfato $\left(\mathrm{PO}_{4}-\mathrm{P}\right)(\mu \mathrm{g} / \mathrm{L})$ & 133,5 & 322,0 & 202,2 & 209,4 & 3472,0 & 58,9 & 1657,7 \\
$\mathrm{DBO}_{5}(\mathrm{mg} / \mathrm{L})$ & 2,5 & 21,0 & 8,7 & 11,3 & 52,4 & 7,2 & 465,1 \\
\hline
\end{tabular}

Tabla 2. Datos obtenidos del metabolismo en la cuenca baja del río Cesar en su máximo estiaje. * = promedio \pm Desviación estándar

\begin{tabular}{|c|c|}
\hline Variables & *Época seca \\
\hline Altitud (m.s.n.m.) & 2257 \\
\hline Sustrato & Arenoso \\
\hline Ancho (m) & 15 \\
\hline Profundidad (m) & 1,85 \\
\hline Velocidad de la corriente $(\mathrm{m} / \mathrm{s})$ & 1,37 \\
\hline Caudal $\left(\mathrm{m}^{3} / \mathrm{s}\right)$ & 2,55 \\
\hline Temperatura ambiente $\left({ }^{\circ} \mathrm{C}\right)$ & $26,4-33,9(29,70 \pm 2,23)$ \\
\hline Temperatura del agua $\left({ }^{\circ} \mathrm{C}\right)$ & $30,6-35,6(32,67 \pm 1,38)$ \\
\hline Oxígeno disuelto (mg/L) & $2,55-5,35(3,58 \pm 0,84)$ \\
\hline Porcentaje saturación de oxígeno (\%) & $26,2-79,2(51,62 \pm 13,36)$ \\
\hline $\mathrm{pH}$ & $7,3-10,8(8,05 \pm 0,69)$ \\
\hline Conductividad $(\mu \mathrm{S} / \mathrm{cm})$ & $469-489(478 \pm 6,25)$ \\
\hline Salinidad (UPS) & $0,1-0,2(0,18 \pm 0,03)$ \\
\hline Nitritos $\left(\mathrm{NO}_{2}-\mathrm{N}\right)(\mathrm{mg} / \mathrm{L})$ & $0,05-008(0,07 \pm 0,01)$ \\
\hline Nitratos $\left(\mathrm{NO}_{3}-\mathrm{N}\right)(\mathrm{mg} / \mathrm{L})$ & $0,39-0,60(0,46 \pm 0,09)$ \\
\hline Fosfatos $\left(\mathrm{PO}_{4}-\mathrm{P}\right)(\mathrm{mg} / \mathrm{L})$ & $11,64-14,52(13,39 \pm 1,25)$ \\
\hline Amonio (mg/L) & 0,4 \\
\hline Alcalinidad (mg/L) & $210-290(250 \pm 38,62)$ \\
\hline $\mathrm{Cl}-\mathrm{a}\left(\mathrm{mg} / \mathrm{m}^{3}\right)$ & 8,058 \\
\hline \multicolumn{2}{|l|}{ Botella oscura } \\
\hline Oxígeno disuelto (mg/L) & $1,4-3,8(2,51 \pm 0,66)$ \\
\hline Porcentaje saturación de oxígeno (\%) & $19,5-64,8(35,07 \pm 10,42)$ \\
\hline $\mathrm{pH}$ & $7,3-10,7(8,01 \pm 0,77$ \\
\hline conductividad $(\mu \mathrm{S} / \mathrm{cm})$ & $483-511(501,72 \pm 8,34)$ \\
\hline \multicolumn{2}{|l|}{ Botella clara } \\
\hline Oxígeno disuelto (mg/L) & $2,8-6(4,12 \pm 0,81)$ \\
\hline Porcentaje saturación de oxígeno (\%) & $39,2-89,1(58,62 \pm 12,98)$ \\
\hline $\mathrm{pH}$ & $7,5-10,8(8,05 \pm 0,68)$ \\
\hline Conductividad $(\mu \mathrm{S} / \mathrm{cm})$ & $475-582(558,08 \pm 41,37)$ \\
\hline
\end{tabular}




\section{Metabolismo}

Los valores mínimos, máximos, promedios y desviación estándar de cada una de las variables medidas durante la curva diaria, se muestran en la tabla 2. En términos generales, en la elaboración de la curva diaria, la cuenca baja del río Cesar presentó un sustrato arenoso con un ancho de $15 \mathrm{~m}$ y una profundidad promedio de $1,85 \mathrm{~m}$, un caudal de $2,55 \mathrm{~m}^{3} / \mathrm{s}$ con una velocidad de corriente de $1,34 \mathrm{~m} / \mathrm{s}$. No se detectaron diferencias significativas entre el OD de las botellas claras y las botellas oscuras, durante el ciclo diario $\left(\mathrm{H}^{\prime}: \mathrm{p}>0,05\right)$, ambas botellas presentaron sus máximos valores de oxígeno disuelto durante las 10:00 $\mathrm{h}$ hasta las 20:00 h. A partir de las 21:00 h los valores de OD de ambas botellas disminuyeron a 1,4 mg/L y 2,8 mg/L, respectivamente. El OD registrado fluctuó, pero, a pesar de ello, se observó una notable disminución a las 21:00 h en ambas botellas (figura 2a).

El \% OD en la botella clara registró el valor más alto $(39,2$ $\%$ ) en comparación con la botella oscura (19,5 \%), durante las 09:00 h (figura $2 \mathrm{~b}$ ). No hubo diferencias significativas del $\mathrm{pH}$ entre las botellas (K-W: p > 0,05). El pH aumentó en las primeras horas del ciclo diario hasta obtener valores máximos de 10,8 en la botella oscura, y en la botella clara
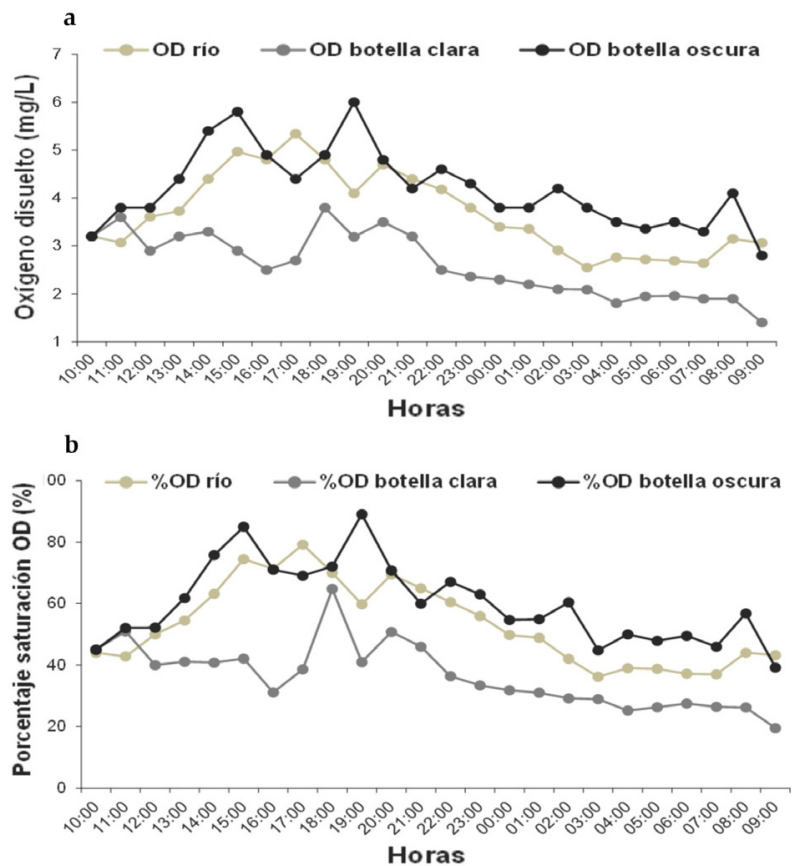

Figura 2. a. Tendencia del OD en la botella oscura y botella clara. b. Tendencia del \% DO en las botellas claras y botellas oscuras, durante la curva diaria. de 10,8 durante las 12:00 h. A partir de esa hora hubo una disminución del $\mathrm{pH}$ desde las 13:00 h hasta las 09:00 h (figura 3a).

La conductividad mostró diferencias significativas entre las botellas $\left(\mathrm{H}^{\prime}: \mathrm{p}<0,05\right)$. Se pudo apreciar que desde las 10:00 h hasta las 14:00 h la conductividad fue similar en ambas botellas; sin embargo, a partir de las 15:00 h, la botella clara aumentó hasta $580 \mu \mathrm{S} / \mathrm{cm}$, valor que se mantuvo constante hasta las 09:00 h (figura $3 \mathrm{~b}$ ) y en la botella oscura se apreció un leve incremento. Finalmente, en relación con el déficit de oxígeno (DO), en la botella oscura fue alto DO $=3,35$ en comparación con la botella clara DO $=2,53$. El comportamiento de ambas botellas evidenció que hay poca producción de oxígeno y mayor consumo del mismo en la cuenca baja del río Cesar, lo que demuestra que en este sistema acuático se consume más oxígeno del que se produce. Por lo tanto, se determinó el metabolismo neto -10 732,23 $\mathrm{O}_{2} / \mathrm{m}^{3}$-h, metabolismo oscuro $-10731,41 \mathrm{O}_{2} / \mathrm{m}^{3}$-h y la producción primaria bruta de la comunidad acuática -10 736,9 $\mathrm{O}_{2} / \mathrm{m}^{3}$-h. El DO para esta época tuvo una tendencia a disminuir (valores negativos) y fluctúo con mayor intensidad entre las 17:00 h y 18:00 h, y menor intensidad entre las 23:00 h a las 01:00 h (figura 4).

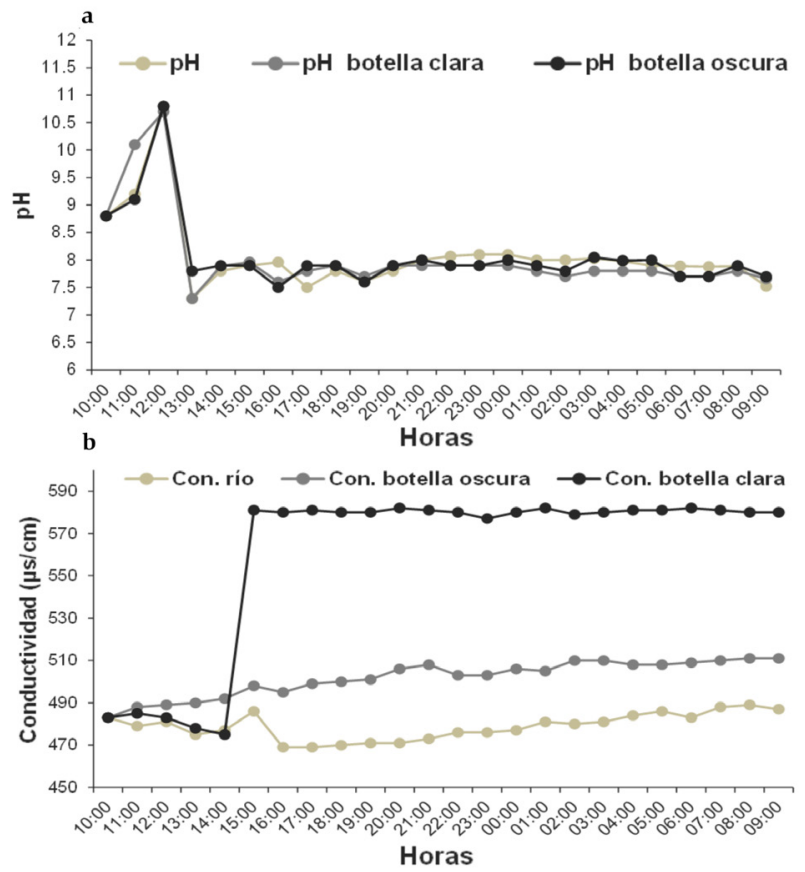

Figura 3. a. Tendencia del pH en las botellas oscuras y botellas claras. b. Tendencia de la conductividad en las botellas oscuras y botellas claras, durante la curva diaria. 


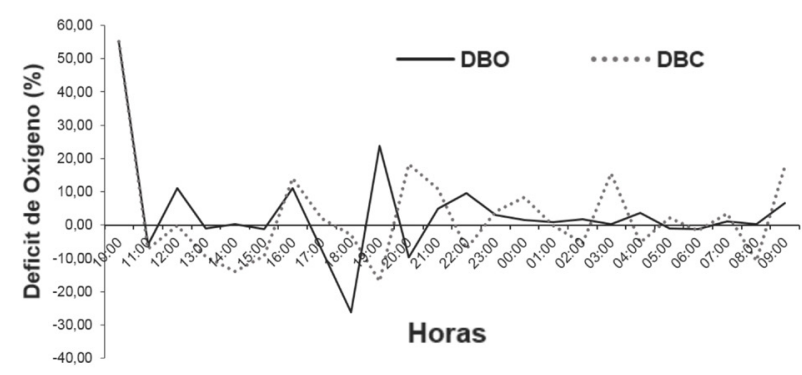

Figura 4. Déficit de oxígeno en la curva diaria de la cuenca baja del río Cesar $(\mathrm{DBC}=$ déficit de oxígeno día y $\mathrm{DBO}=$ déficit de oxígeno noche).

\section{Discusión}

Los valores de temperatura observados en la cuenca baja del río Cesar durante la época seca, indican el comportamiento térmico caracterizado por aguas cálidas con altas temperaturas correspondientes con la época de sequía y zonas con poca vegetación (arbustivas), limitando la sombra sobre el espejo de agua, lo que probablemente contribuye a la radiación directa del sol, y con esto al incremento de la temperatura en este tramo del río. Las concentraciones bajas de OD se atribuyeron a la zona baja del río, donde la temperatura es mayor con respecto a los otros tramos. Otras posibles fuentes que influyeron en los valores de oxígeno son la acumulación de materia orgánica, aumento de nutrientes, aguas residuales de las comunidades ribereñas y vertimientos procedentes de la ganadería, agricultura y zonas industriales (Roldán y Ramírez, 2008).

Estos vertimientos se acumulan con el tiempo en el fondo de las aguas contaminantes y, al llegar la sequía, son mezclados y resuspendidos de la columna de agua, aumentando las partículas en suspensión, sedimento, la turbidez y los SST (De la parra et al., 2017). La cuenca baja del río Cesar es un ecosistema ligeramente básico, condición propia de un sistema tropical (Roldán y Ramírez, 2008). Se pudo observar una $\mathrm{DBO}_{5}$ relacionada al máximo estrés hídrico.

Todas las características fisicoquímicas mencionadas anteriormente en este sistema lótico son soportadas y corroboradas en la teoría del río continuo (Vannote et al., 1980), y en investigaciones relacionadas con la limnología Neotropical de Dodds (2007); Dodds y Cole (2007) y Roldán y Ramírez (2008), donde se manifiesta que cada tramo o sector del río tiene condiciones diferentes ya sea por cambios sucesionales o graduales de las comunidades y las variables ambientales. El caudal, al bajar los niveles 21 de agua, disminuyó en comparación con reportes en épocas de lluvia (Rivera y Donato, 2008) lo que favorece la acumulación de materia orgánica provenientes del área terrestre, incrementándose los nutrientes en el agua. Asimismo, las zonas secas del lecho del río, durante el período de sequía concentran la materia orgánica que contribuye al aumento del sedimento, que es arrastrado posteriormente cuando aumenta el caudal. Descripciones similares han sido observadas en otras cuencas de sistemas naturales tropicales (Rivera y Donato, 2008).

Los altos valores registrados de conductividad eléctrica posiblemente estén asociados a la resuspensión de materiales del fondo del agua, que a su vez entran a jugar un papel activo en la dinámica de nutrientes en el sistema. Otra posible explicación puede estar relacionada con factores como la geoquímica del terreno, la erosión del cauce, el arrastre de sedimentos por la entrada de caños o canales al sistema, la actividad agrícola y los vertimientos domésticos sin previo tratamiento, por presencia de algunas lluvias locales o sectorizadas (Fontalvo y TamarisTurizo, 2018).

La cuenca baja del río presentó un estado trófico relacionado con la materia orgánica, además de todas las variables antes discutidas y mencionadas. La cuenca baja es clasificada como eutrófica, lo que conlleva a un predominio de la producción y, además, junto con la Cl-a del fitoperifiton $8,05 \mathrm{mg} / \mathrm{m}^{3}$ (tabla 3), se le suma un carácter mesotrófico, rango establecido por sistemas de calificación trófica (OECD, 1982).

El metabolismo en los ecosistemas acuáticos se refiere a procesos de producción, consumo y descomposición. Estos procesos ocurren de forma simultánea, balanceada y sincronizada (Oseguera et al., 2015). Sin embargo, cuando el equilibrio es alterado dada a la contaminación por materia orgánica, procesos de descomposición y fotosíntesis son acelerados en el ciclo día-noche, causando un desbalance en la estabilidad del ecosistema y las comunidades que allí habitan (García-Alzate et al., 2010; Oseguera et al., 2015). Por ello, el estado trófico en estos sistemas acuáticos puede determinarse a partir de la actividad metabólica.

La cuenca baja del río Cesar se clasifica como un ecosistema eutrófico-heterotrófico, donde la respiración excede la producción, relación producción/respiración $(\mathrm{P} / \mathrm{R})<1$. Esto sugiere que la producción autóctona es completada por aportes de materia orgánica alóctona procedente de los ecosistemas terrestres, por tanto, se considera que la comunidad biótica depende de la materia orgánica que llega del lavado de la cuenca y de los materiales arrastrados por 
escorrentía, además de las variables fisicoquímicas medidas durante la época, que corroboran el estado de eutrofización que presenta el cuerpo de agua. Es importante resaltar en esta investigación la sectorización del río, pues se presentan condiciones diferentes ya sea por cambios continuos de las comunidades o cambios fisicoquímicos, además de que estas condiciones se ven afectadas por variaciones climáticas y fenómenos ambientales que se observan en la actualidad (Dodds y Cole, 2007; Roldán y Ramírez, 2008).

\section{Agradecimientos}

Al programa de Maestría en Biología y Grupo de Investigación Biodiversidad del Caribe colombiano de la Universidad del Atlántico. A la Corporación Autónoma Regional del Cesar (CORPOCESAR) por la financiación de la investigación, convenio $\mathrm{N}^{\circ}$ 19-7002-0-2013 del 2 de mayo del 2013 proyecto: Plan de Ordenamiento del Recurso Hídrico del Río Cesar. A la beca Apoyo Institucional de Maestría y Doctorado de la Universidad del Atlántico. A COLCIENCIAS, (Convocatoria No. 727 de 2015. Res. 513, julio de 2015). A Jenny Morales, Katy Retamoza y Renzy Medrano por su colaboración. A todos los habitantes de los municipios ribereños del río Cesar por sus conocimientos empíricos.

\section{Referencias}

APHA. 2005. Standard Methods for the Examination of Water and Wastewater, American Public Health Association, EE.UU.

Bunn, S., Davies, P. y Mosisch, T. 1999. Ecosystem measures of river health and their response to riparian and catchment degradation. Freshwater Biology 4 (12): 333-345.

De la parra, A., García-Alzate, C., Rodelo, K. y Gutiérrez, L. 2017. Fitoperifiton composition and structure of the middle and lower basin of the river Cesar, Cesar DepartmentColombia. MVZ Córdoba 22(2): 5938-5950.

Dodds, W. 2006. Eutrophication and trophic state rivers and streams. Limnology Oceanography 51: 671-680.

Dodds, W. 2007. Trophic state, Eutrophication and nutrient criteria in streams. Trends in Ecology and Evolution 22 (12): 669-676.

Doods, W. y Cole, J. 2007. Expanding the concept of trophic state in aquatic ecosystems: It's not just the autotrophs. Aquatic Sciences 69 (4): 427-439.

Donato, J., Abuhatab, Y. y Sabater, S. 2014. Epilithic biofilm metabolism during the high water flow period in an Andean Neotropical stream. Hydrobiologia 728 (1): 41-50.
Fontalvo, F. y Tamaris-Turizo, C. 2018. Calidad del agua de la parte baja del río Córdoba (Magdalena, Colombia), usando el ICA-NSF. Intropica 13(2): http://dx.doi. org/10.21676/23897864.2510.

García-Álzate, C., Román-Valencia, C., Vanegas-Ríos, A., y Arcila-Mesa, D. 2007. Análisis fisicoquímico y biológico comparado en dos quebradas de alta montaña Neotropical. Revista de Investigaciones Universidad del Quindio 17: 57-80.

García-Alzate, C., Román-Valencia, C., Lopera, D., González, M. y Simunovic, M. 2008. Physico-chemical and Biological variables of San José creek, Otun river drainage/upper Cauca, Colombia. Revista de Investigaciones Universidad del Quindio 18 (240): 38-48.

García-Alzate, C., Román-Valencia, C., Taphorn, D. y González, M. 2010. Physicochemical and Biological Characterization of de Roble River, Upper Cauca, western Colombia. Museo Argentino Ciencias Naturales 12(1): 4-16.

Gutiérrez, J. 2006. Caracterización del metabolismo y de la oferta de recursos de materia orgánica para la fauna de macroinvertebrados bentónicos en una quebrada de montaña de orden menor. Tesis Doctoral, Universidad Nacional de Colombia, Bogotá, Colombia.

Hammer, Q., Harper, D.A. y Ryan, P.D. 2001. PAST: Paleontological Statistics software package for education and data analysis. Paleontología Electrónica 4(1): 1-9.

IDEAM, 2006. Aprovechamiento y protección integral del agua subterránea en los valles del río Cesar y valle del río Magdalena. Informe final del convenio 047 de 2004.

Montoya, Y. y Aguirre, N. 2009. Estado del arte de la limnología de lagos de planos inundables (ciénagas) en Colombia. Gestión y Ambiente 12 (3): 85-106.

Montoya, Y. y Aguirre, N. 2013. Estado del arte del conocimiento del fitoperifiton en Colombia. Gestión y Ambiente 16(3): 91-117.

Mulholland, P., Fellows, C., Tank, J., Grimm, N., Webster, J., Hamilton, S., Marti, E., Ashkenas, L., Bowden, W., Doods, W., McDowell, W., Paul, M. y Peterson, B. 2001. Inter-biome comparison of factors controlling stream metabolism. Freshwater Biology 46 (11): 1503-1517.

OECD (Organización para la Cooperación y el Desarrollo Económico). 1982. Eutrophication of Waters. Monitoring, Assessment and Control. Cooperative Programmers on Monitoring of Inland Waters (Eutrophication Control), Environment Directorate, OECD Paris, Final Report. France. 
Oseguera, L., Alcocer, J., Quintero, V. y Einye, Y. 2015. Metabolismo lacustre de un lago tropical profundo: ifuente o sumidero de carbono?. Hidrobiológica 25(3): 391-399.

Ramírez, A. 2001. Algas del perifiton, productividad y materia orgánica de un río de alta montaña tropical. Tesis de pregrado, Pontificia Universidad Javeriana, Bogotá, Colombia.

Rivera, C. y Donato, J. 2008. Influencia de las variaciones hidrológicas y químicas sobre la diversidad de diatomeas bénticas. Donato, J. Editor. Ecología de un río de montaña de los Andes Colombianos (Río Tota, Boyacá). Colombia.
Roldán, G. y Ramírez, J. 2008. Fundamentos de Limnología Neotropical. Segunda Edición. Editorial Universidad de Antioquia. Medellín.

Vannote, R., Minshall, G., Cummins, K., Sedell, J. y Cushing, C. 1980. The River Continuum Concept. Canadian journal of fisheries and aquatic sciences 37(1): 130-137.

Wetzel, R. y Likens, G. 2000. Limnological analyses, 3 Ed Editorial Springer-Verlag New York.

Citar como: De la parra, A. y García-Alzate, C. 2019. Metabolismo de un tramo en la cuenca baja del río Cesar, departamento del Cesar, en una época de sequía, Colombia. Intropica 14(1): 16-23. DOI: http://dx.doi.org/10.21676/23897864.2719. 